\title{
Research On Real-time Processing System Of Hyper-spectral Image Based On FPGA
}

\author{
YuanBo $^{1,2}, \mathrm{Hu}_{\text {Bingliang }}{ }^{1}$, GaoJiarui $^{1}$ \\ 1. Xi'an Institute of Optics and Precision Mechanics, Chinese Academy of Science, Xi'an \\ 710119, China
}

2.Graduate School of the Chinese Academy of Sciences, Beijing 100039, China

Keyword: Hyper-spectral image , PCA, System Generator , FPGA

\begin{abstract}
The real-time is an importance on judging the performance of the image processing system . The instantaneity means the system must have the reaction in the definitive time .In this research , the time in disposing an image must be limited in millisecond from receive the image to operate and report the information . So this customized image processing system should have the enough operation ability in hardware, combine the software and related algorithm to guarantee the real-time of the process .
\end{abstract}

\section{Introduction}

Most of the image process system can fulfill the real-time demand in low-resolution, but cannot fulfill in the high-resolution . Hyper-spectral image means high-resolution、 high-bandwidth data , the processing speed is always $\mathrm{nG}$ bit/s, the traditional serial design cannot meet the requirement , the characteristic in parallel processing let FPGA provide new ways to solve this problem .

\section{PCA}

PCA as Principal Components Analysis, the principle theory is let the previous variable reconstitute to several unconcerned aggregative variable and wipe off fewer ones according the actual requirement at the same time, response the previous variable message as many as possible . This method can be used in dimensionality reduction and feature extraction .

Assume $\mathrm{X}$ is an $\mathrm{m}^{*} \mathrm{n}$ matrix and constitute by the sample data .m represent attribute dimension , $\mathrm{n}$ represent sample number. Throw linear transformation make $\mathrm{X}$ matrix into $\mathrm{Y}$ matrix , let the covariance matrix of $\mathrm{Y}$ to diagonal matrix , thus $\mathrm{Y}$ considered as the principle component of $\mathrm{X}$.

The linear transformation formula from $\mathrm{X}$ to $\mathrm{Y}: \mathrm{Y}=\mathrm{PX}$

Covariance of $X$ and $Y: C_{X}=\frac{1}{n} X X^{T} C_{Y}=\frac{1}{n} Y Y^{T}$

The relation between $C_{Y}$ and $: C_{X}$

$$
\begin{aligned}
& C_{Y}=\frac{1}{n} Y^{T} \\
= & \frac{1}{n} Y^{T} \\
= & \left.P\left(\frac{1}{n} X^{T}\right)(P X)^{T}\right) P^{T} \\
C_{Y} & =P_{X} P^{T}
\end{aligned}
$$

$\mathrm{C}_{\mathrm{X}}$ is symmetric matrix , so eigenvalue decomposition make $\mathrm{X}$ into diagonal matrix ,

$=\mathrm{P}\left(\mathrm{E}^{\mathrm{T}} \mathrm{DE}\right) \mathrm{P}^{\mathrm{T}}$

$$
\mathrm{C}_{\mathrm{Y}}=\mathrm{PC}_{\mathrm{X}} \mathrm{P}^{\mathrm{T}}
$$

$=\left(\mathrm{PP}^{-1}\right) \mathrm{D}\left(\mathrm{PP}^{-1}\right)$

$\mathrm{C}_{\mathrm{Y}}=\mathrm{D}$

Above the formula : $\mathrm{P}=\mathrm{E}^{\mathrm{T}}$ 
$\mathrm{D}$ is diagonal matrix constitute by the eigenvector of $\mathrm{C}_{\mathrm{X}} . \mathrm{P}$ is linear narrative, every line of $\mathrm{P}$ is the diagonal matrix of $\mathrm{C}_{\mathrm{X}}$, and $\mathrm{P}$ is orthogonal matrix .

So , work out $\mathrm{P}$ can get the principal component matrix .

Above all there are about three steps to bring out the principal component of Hyper-spectral image :

1、 minus the average value

Work out the average value of all spectral coverage, minus the one of the original image .

2、 work out the variance

Transform the three-dimensional image into two dimension ( spatial dimension and spectrum dimension ), get a $\mathrm{N}^{*} \mathrm{P}$ matrix named I , work out the covariance of I , form a $\mathrm{N}^{*} \mathrm{~N}$ matrix .

3、 work out the eigenvalue of $\mathrm{N}^{*} \mathrm{~N}$ matrix

The eigenvalue is the principal component of the image, at last reconfiguration the image .
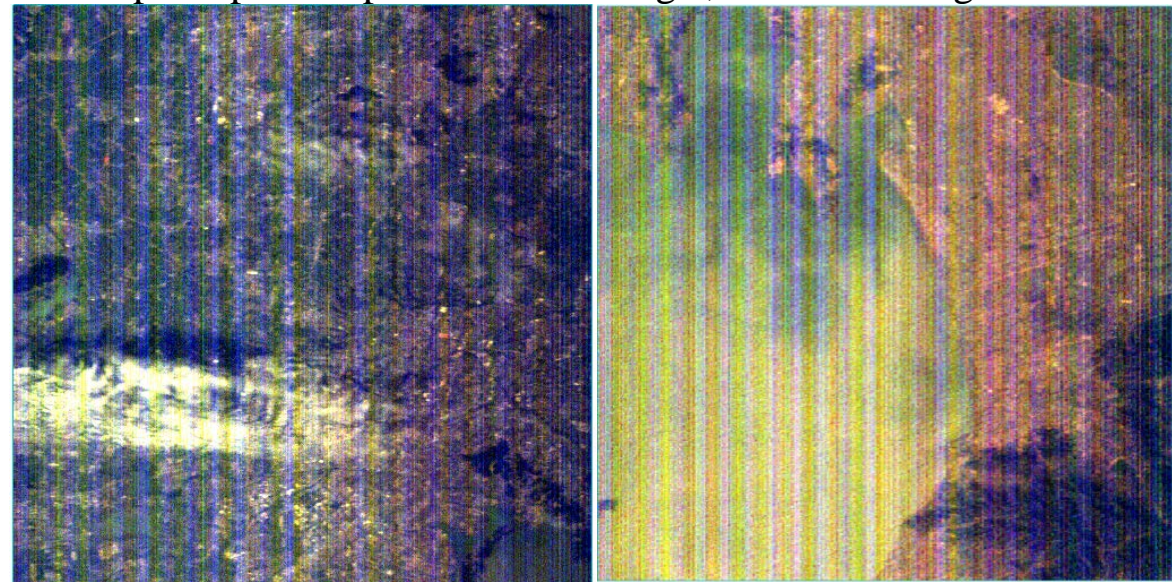

The left image is not disposed, the right one is disposed . In the right one , it can recognize the lake and lakeshore obviously. So it have an impressive effect in using Matlab to analysis the principal component .

\section{Use the System Generator as an instrument to generate Verilog code}

1、System Generator can imaging modeling the hardware system in need, under the condition of Simulink the modeling number system can translate into FPGA project .It reduce the time of logic design and the hardware technological process, and lessen the probability of error .And use Sys-Gen to emulation the system can simplify the complex test-bench ; What's more , Sys-Gen hardware union simulation function can let the FPGA act as part of the system design , make the large-scale parallel computing which improved come true , and then can accelerate the simulation speed of other section .

Above all there are about five steps :

(1) Describe the arithmetic model with mathematical method

(2) Set up and simulate the system model , in the beginning use double precision

(3) Make the double precision arithmetic into fixed point

(4) Start System Generator , generate Verilog code and other files

(5) Simulate and debugging

2、 System Generator ordinary model 


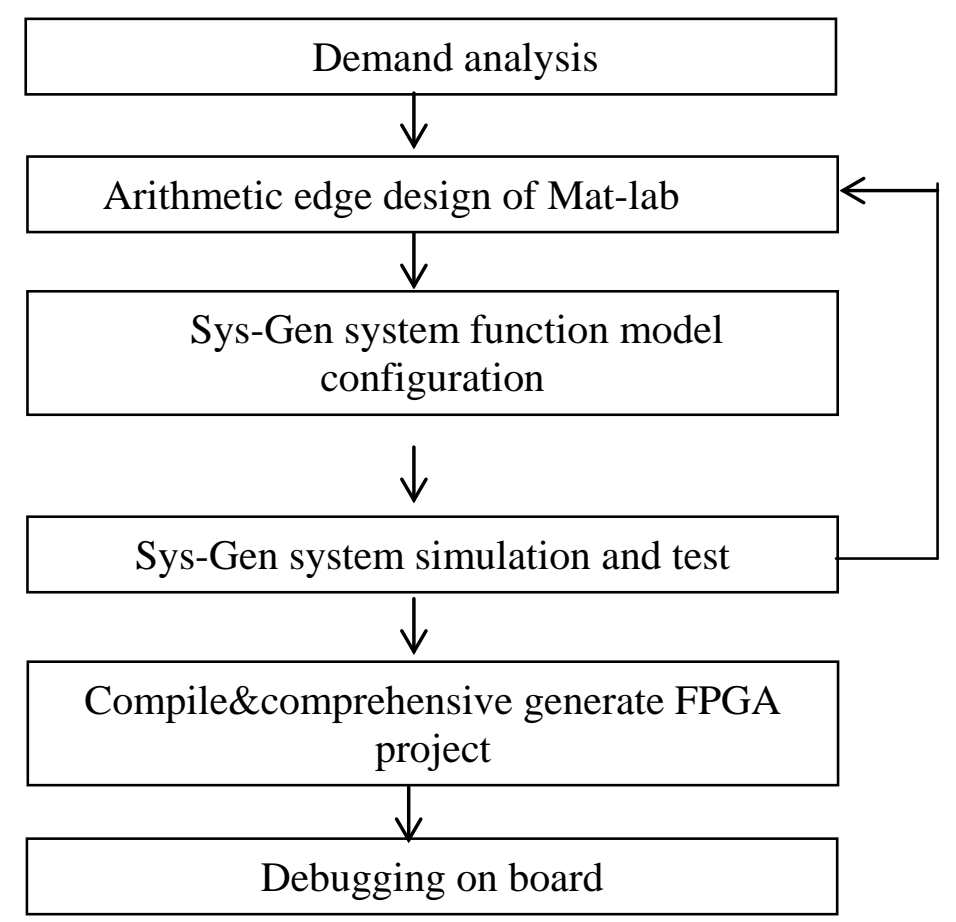

\section{The principal component analysis board of Hyper-spectral image}

1、Software

As to the 1000fps image , the processing speed of hardware must be under millisecond level . So ,the logic design part of FPGA in the arithmetic, the write and read design of data flow can ensure the demand of real-time .

2、 hardware

Because of the succession pattern of DSP , the speed of data processing is slow , and it lack the characteristic of real-time, which restrict the apply of DSP that high demand in speed and capacity .

FPGA constitute with a large number logic macro-cell that linked by switch network , internal logic cell and switch network are all programmable , throw the configuration these cell can form different hardware structure ,and achieve different function .

(1) The operating mode of DSP

Data In

256 loops needed

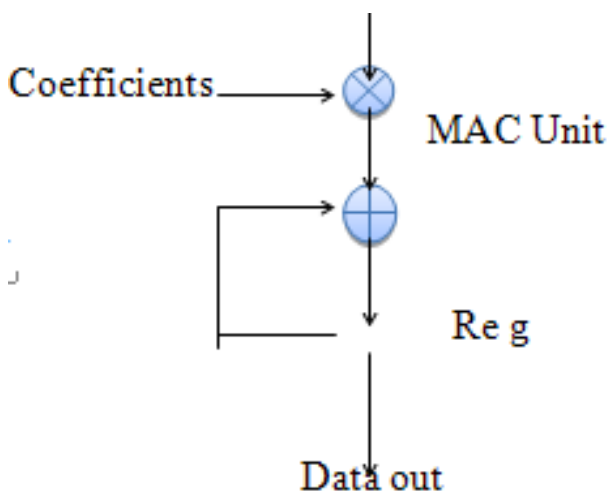

to process samples

For $1 \mathrm{G}$ data , 256 cycles, it can transmit 4M data in every cycle .

(2) The pipeline model of FPGA

$$
\frac{1 \mathrm{GHz}}{256 \text { clock cycles }}=4 \mathrm{MSPS}
$$

FPGA-based DSP - Parallelism 


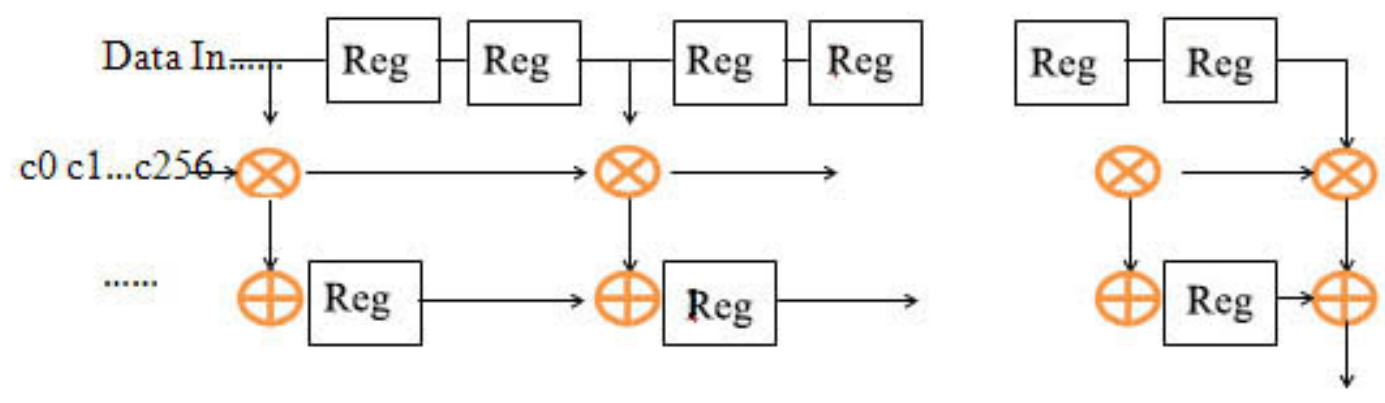

Data out

$$
\frac{500 \mathrm{MHz}}{1 \text { clock cycles }}=500 \mathrm{MSPS}
$$

So , the transmit speed can reach 500MSPS in theory with the parallel processing of FPGA, which make the real-time transmit came true .

3、 The block diagram of image processing

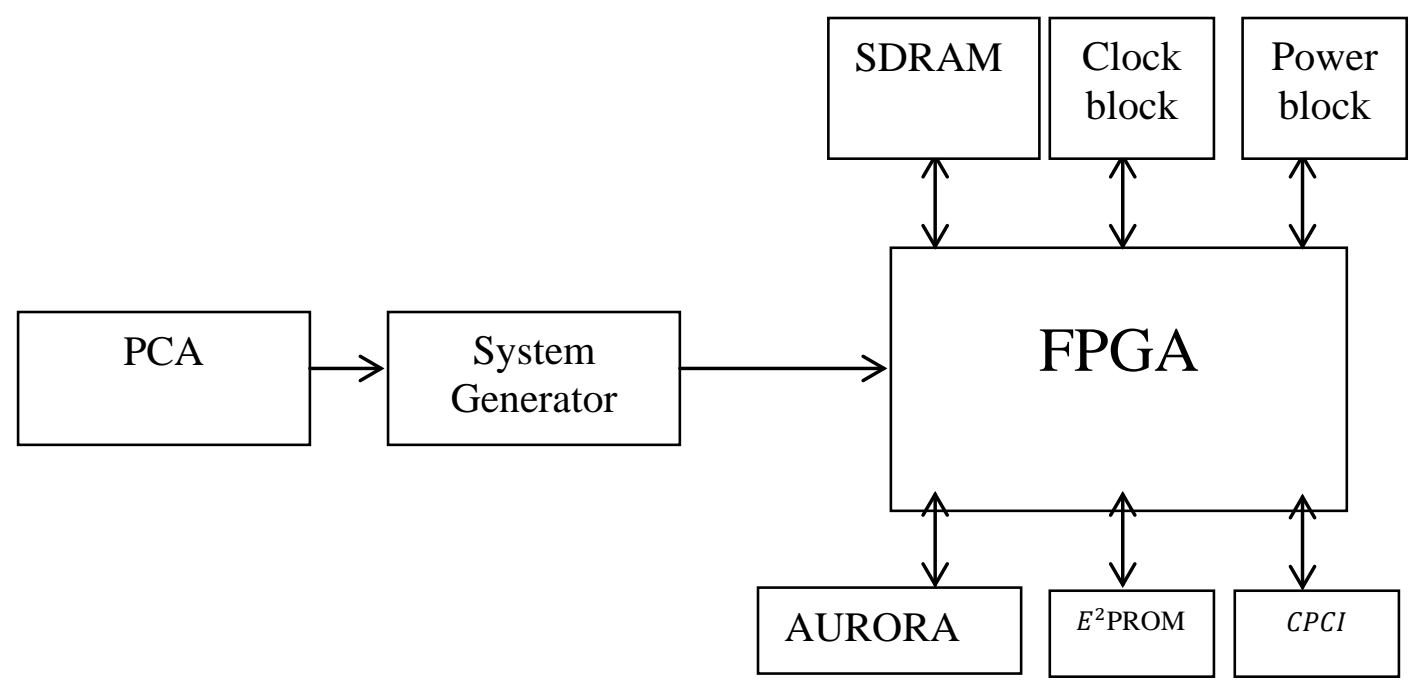

\section{Spectral inversion processing card-time distribution of real-time processing model}

Pipeline design of FPGA

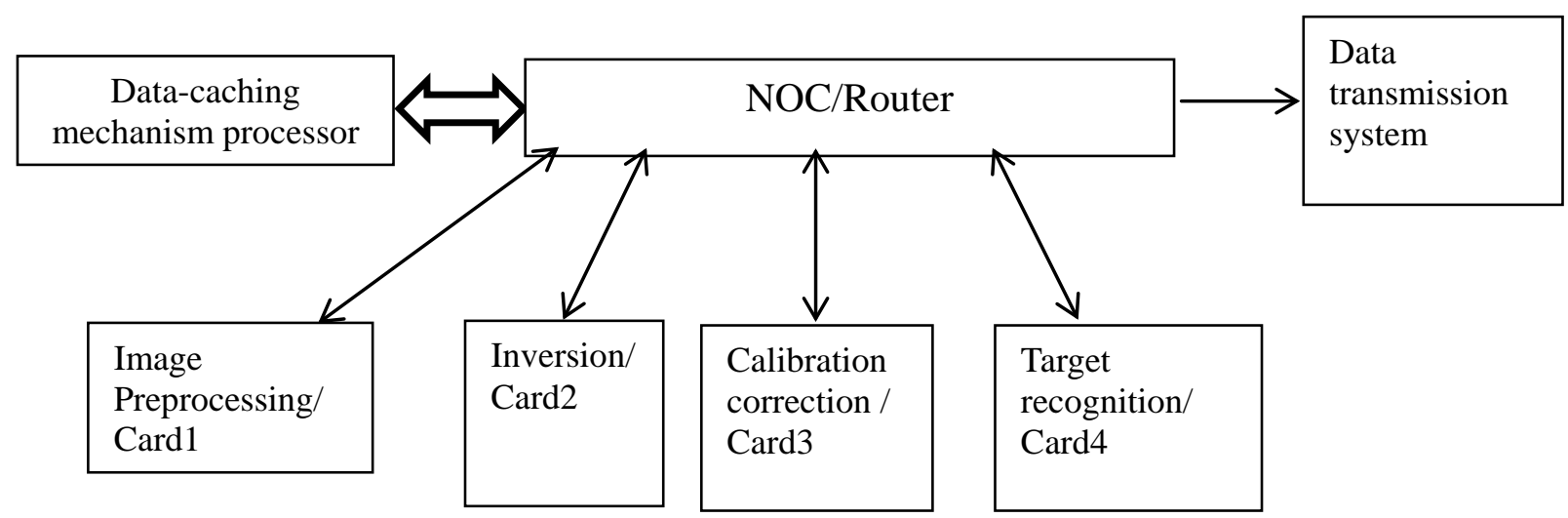

Data volume of the original image : 4096*256*12bit*266fps $=3.35 \mathrm{Gbps}$

Space between two frame : 266fps, equal to $3.76 \mathrm{~ms}$

Time distribution of the Spectral inversion processing card :

Core processing device of FPGA of every block, use the $125 \mathrm{MHz}$ clock and 5Gbps SRIO connector

By analysis, the time of full algorithm processing is $9.2 \mathrm{~ms}$ : 


\begin{tabular}{|l|l|l|l|l|}
\hline Card & $\begin{array}{l}\text { Image } \\
\text { Preprocessing }\end{array}$ & Inversion & $\begin{array}{l}\text { Calibration } \\
\text { correction }\end{array}$ & $\begin{array}{l}\text { Target } \\
\text { recognition }\end{array}$ \\
\hline time & $1.8 \mathrm{~ms}$ & $3.5 \mathrm{~ms}$ & $2.9 \mathrm{~ms}$ & $1 \mathrm{~ms}$ \\
\hline
\end{tabular}

According to the form , the time of the system will spend 3.5ms when use the pipeline work pattern .

\section{Summary}

In this paper ,work out the principal component analysis of Hyper-spectral image in the Mat-lab platform, and get the major message of the image with an impressive effect after processing . Graphical model the hardware system in need by System Generator , the modeling number system transform FPGA project under the Simulink condition, finish the transform from Mat-lab code to FPGA code . According the pipeline character of FPGA design the read and write of the data flow of the system to make sure the demand of real-time ,achieve live transmission of the Hyper-spectral image in the end .

\section{References}

[1] Hua Yang, The research of real-time image acquisition \& processing system based on FPGA , 2009

[2] YongliWang,The study and design of real-time iamge processing platform based on FPGA , 2011

[3] BinkaiNiu ,Research on Design Method for Digital Signal Processing System Based on System Generator , 2009

[4] PengZhao , The Hardware Design And Implementation Of Real-time Image Processing System

[5] HuanZhang, Design of experimental platform for real-time image processing based on FPGA 2014

[6] XiangmeiKong , Design of real-time image noise reduction system based on FPGA, 2009

[7] Li Yunfei , The implementation of digital down_conversion based on the system generator , 2008 